\title{
Efficacy of empirical antibiotic prescription for the treatment of community acquired pneumonia
}

\author{
S Akhter ${ }^{1}$, SM Kamal ${ }^{2}$, MF Islam ${ }^{3}$, MKA Mollick ${ }^{4}$, MN Islam $^{5}$
}

\begin{abstract}
Background: Community acquired pneumonia (CAP) is a leading cause of morbidity and mortality in Bangladesh. There is difficulty in identification of the causative organism due to lack of facility of sputum culture and sensitivity test in many hospitals of Bangladesh. So targeted anti microbial therapy is not possible and empirical antibiotic prescription is done. The choice of empirical selection of antibiotic should be the best effective drug for the treatment of CAP.

Objective: To assess the efficacy of selected empirical antibiotic selection for the treatment of CAP.

Methods: This prospective observational study involves 82 patients with CAP admitted in medicine ward of Khulna medical college hospital, Khulna, Bangladesh. Patients were selected by inclusion and exclusion criteria. Diagnosis of CAP was confirmed by chest $x$-ray. Empirical selection of antibiotic was based on clinical experience. Most of the patients were treated with combined antibiotic except a few with monotherapy. Evaluation of the empirical choice of ant biotic was done by the outcome. Data of different parameter were recorded during study in preformed proforma. Statistical analysis was done by using SPSS.
\end{abstract}

Result: Out of 82 patients 62 (76\%) were male and 20 (24\%) were female. Mean age was 54.4 ( \pm 9.6) years. Duration of antibiotic treatment was 7 days. No drug related adverse effect was observed. One patient developed paraneumonic effusion and none of the patient developed lung parenchymal damage or lung abscess. All the patient recovered completely.

Conclusion: CAP is treated in many hospitals of Bangladesh by selecting the antibiotic empirically. Improvement of hospital laboratories to perform sputum for culture and sensitivity test is necessary for selecting an effective anti biotic.

Key wards: Community acquired pneumonia, Empirical, Antibiotic.

\section{Introduction}

Community acquired pneumonia (CAP) is the pneumonia occurring outside the hospital or in long term care facility. It causes morbidity and mortality world wide. Adverse cardiovascular events can also occur. Patients present with cough, fever, chills, rigor, fatigue, dyspnoea and pleuritic chest pain. Assessment for the need of hospitalization is done by using the pneumonia severity index, CURB 65 score system combined with clinical judgment. CURB 65 scoring is done by calculating scores on Confusion (mini mental state score <8). blood Urea level (> $7 \mathrm{mmol} / 1)$. Respiratory rate ( $>30 /$ minute), Blood pressure (systolic blood pressure $<90 \mathrm{mmHg}$ and diastolic blood pressure $<60 \mathrm{mmHg}$ ) and Age $>65$ years. One point score for each character and total point score is 5. Patient having score $0-1$ is recommended for outpatient care, score 2 is recommended for in patient ward care and score ${ }^{3}$ 3 is recommended for in patient intensive care unit (ICU) care. Incidence of hospitalization is high among elderly adults.1 Diagnosis is done by history, clinical features and by some

1. Shamima Akhter M.Phil, Assistant professor, Dept of Pharmacology, Khu1na Medical College. (E-mail: shamimakhulna94@gmail.com)

2. SM Kamal FCPS, Professor, Department of Medicine, Khulna Medical College, Khulna.

3. Md Farhadul Islam MBBS, Registrar, Department of Medicine. Khulna Medical College Hospital, Khulna.

4. Md Khosrul Alam Mollick MD, Associate Professor, Department of Respiratory Medicine, Khulna Medical College, Khulna.

5. Md Nazrul Islam FCPS, Junior Consultant, Department of Medicine, Khulna Medical College Hospital, Khulna. 
investigations such as chest $\mathrm{x}$ ray, complete blood count, blood sugar, blood for culture and sensitivity test and sputum for culture and sensitivity (sputum for $\mathrm{c} / \mathrm{s}$ ) test. ${ }^{2}$ Despite advanced diagnostic tests no organism can be detected in some cases and respiratory viruses are detected in few cases. ${ }^{3}$

Empirical selection of antimicrobial for the treatment is the cornerstone of management. To reduce the misuse of antibiotic and to avoid the antibiotic resistance an effective antibiotic selection is essential. Empirical selection means choice originating in or based on clinical experience. Follow up after the start of antibiotic is important. ${ }^{4}$ Empirical dual antibiotic therapy consisting of Beta-lactum plus macrolide (clarithromycin) or fluroquinolone (levofloxacin) or doxycycline initiated within 4 to 8 hours of hospitalization was associated with lower mortality.5,6 Switch over from parenteral antibiotic to oral antibiotic can be done after improvement of symptoms when patient is afebrile and able to tolerate oral medication. ${ }^{7}$

CAP in out patient department can be treated with monotherapy using macrolide (clarithromycin) or doxycycline. 8 Patients having co-morbidity like chronic obstructive pulmonary disease (COPD) can be treated with respiratory fluroquinolone (levofloxacin or gemifloxacin or moxifloxacin) or an oral Beta-lactum plus a macrolide (clarithromycin) by empirical selection. Severe CAP admitted in ICU can be treated with a Beta-lactum plus a macrolide (azithromycin) or a respiratory fluroquinolone. Patients with the risk of having pseudomonas infection can be treated with lactum antibiotic (such as piperacillin / tozabactum, imipenem / cilastatin, meropenem, doripenem or cefepime) plus an aminoglycoside or an anti pseudomonal fluroquinolone (levofloxacin or ciprofloxacin). Those with the risk of methicillin resistant staphylococcus aureus infection can be treated with vancomycin or linezolid.9,10 Anti pneumococcal or anti influenza vaccination can be done in elderly patients to prevent CAP by these organisms. ${ }^{11}$

The aim of this study was to emphasize on the choice of the empirical selection of antimicrobial. The choice should be the best possible drug for the treatment of CAP and individualized for each patient in terms of efficacv of the the antibiotic either as single agent or in combination treatment.

\section{Materials and Methods}

This prospective observational study was carried out in the medicine ward of Khulna medical college hospital, Khulna, Bangladesh, during a period of 6 months from 1st August 2018 to 31st January 2019. Ethical clearance was taken from the Ethical review committee of Khulna medical college, Khulna. Total 82 adult male and female patients with community acquired pneumonia (CAP) were enrolled in this study. Patients were selected by inclusion and exclusion criteria. Patients with pneumonia occurring outside the hospital were included in this study. Patients with pulmonary tuberculosis, pleural effusion due to tuberculosis, bronchogenic carcinoma, heart failure, renal failure and pregnant patients were not included in this study. Informed written consent was taken from each patient before their enrollment. History and physical examination was completed in each patient. CAP was defined as the presence of acute illness with two or more of the symptoms and signs of lower respiratory tract infection such as fever, cough, sputum, dyspnoea, chest pain, features of consolidation on examination of chest and presence of radiological features of consolidation in chest $x$ ray. CAP severity index CURB 65 scoring was done by calculating scores on Confusion (mini mental state score < 8) blood Urea level (> $7 \mathrm{mmol} / 1)$, Respiratory rate ( $>30 /$ minute), Blood pressure (systolic blood pressure $<90 \mathrm{mmHg}$ and diastolic blood pressure $<60 \mathrm{mmHg}$ ) and Age $>65$ years. One point score for each character and total point score is 5. Patient having score $0-1$ is recommended for out patient care, score 2 is recommended for in-patient ward care and score ${ }^{3}$ 3 is recommended for in patient intensive care unit (ICU) care. Pulse, temperature and chest $\mathrm{x}$ ray findings were recorded in each patient. Presence of co-morbid conditions such as asthma, chronic obstructive pulmonary disease (COPD), hypertension, diabetes mellitus, use of steroid or other immunosuppressive drugs and smoking habit were determined and recorded from history and treatment documents. Sputum character whether rusty or purulent was observed and recorded. Sputum culture and sensitivity test was not done due to lack of facility. Antibiotic was selected for each' patient on empirical basis from the knowledge of previous experience and observation in treating the CAP patients. Antibiotics used were inj. Ceftriaxone $2 \mathrm{gm}$ iv daily in $12(15 \%)$ patients. inj. Ceftriaxone $2 \mathrm{gm}$ iv daily plus oral Clarithromycin $250 \mathrm{mg} 12$ hourly in 44 
(55\%) patients, oral Co-amoxiclav $375 \mathrm{mg} 8$ hourly plus oral Clarithromycin $250 \mathrm{mg} 12$ hourly in 12 $(15 \%)$ patients, inj. Ceftriaxone, 2 gm iv daily plus oral Cloxacillin $500 \mathrm{mg} 6$ hourly in 10 (12\%) patients, inj. Ceftriaxone $2 \mathrm{gm}$ iv daily plus oral Linezolid $600 \mathrm{mg} 12$ hourly in $2(2 \%)$ patients and inj. Meropenem $500 \mathrm{mg}$ iv 8 hourly in $2(2 \%)$ patients. Antibiotic treatment was continued for 7 days. No drug related adverse effect was observed in any patient. Treatment outcome was observed and recorded by follow up of the clinical features and chest $x$ ray findings done after 7 days. Chest $\mathrm{x}$ ray after 7 days reveals complete resolution of the lung consolidation. There was no lung parenchymal damage or abscess formation. Evaluation of the empirical use of antibiotic in individual patient was done by the outcome. Data of different parameter were recorded during study in preformed proforma. Statistical analysis was done by using SPSS.

\section{Result}

82 patients were enrolled in this study. 62 (76\%) were male and 20 (24\%) were female. Age was from 21 to 82 years. Mean age was $54.4 \pm 9.6$ years. Highest number of patients-20 (25\%) were in 31 to 40 years age group and $22(27 \%)$ patients were of $>65$ years old. (Table-I)

Table I

Age and sex distribution of the patients

\begin{tabular}{|c|c|c|c|c|}
\hline \multirow{2}{*}{$\begin{array}{l}\text { Age group } \\
\text { in years }\end{array}$} & \multicolumn{2}{|c|}{ Number } & Number & \multirow[t]{2}{*}{ Mean age } \\
\hline & Male & Female & Total & \\
\hline $21-30$ & 6 & 2 & 8 & 9 \\
\hline $31-40$ & 20 & 0 & 20 & 25 \\
\hline $41-50$ & 8 & 8 & 16 & 20 \\
\hline $51-60$ & 6 & 2 & 8 & 9 \\
\hline $61-70$ & 10 & 2 & 12 & 15 \\
\hline $71-80$ & 10 & 6 & 16 & $2054.4 \pm 9.6$ \\
\hline $81-90$ & 2 & 0 & 2 & 2 \\
\hline Total & 62 & 20 & 82 & 100 \\
\hline
\end{tabular}

$28(34 \%)$ patients were without any co-morbidities $54(66 \%)$ patients had co-morbidities such as diabetes mellitus (8-10\%), immunosuppression due to steroid intake (4-4\%), COPD (6-7\%), asthma (8-10\%), Parapneumonic effusion (1-1\%), smoking habit (15-19\%) and hypertension (12 15\%) (Table-II).
Table II

Co-morbidities of the patients

\begin{tabular}{lcc}
\hline Co-morbidity & No & $\%$ \\
\hline Chronic obstractive & 6 & 7 \\
pulmonary disease & & \\
Asthma & 8 & 10 \\
Smoking & 15 & 19 \\
Diabetes mellitus & 8 & 10 \\
Immunosuppression & 4 & 4 \\
Hypertension & 12 & 15 \\
Parapneumonic effusion & 1 & 1 \\
No Co-morbidity & 28 & 34 \\
\hline Total & 82 & 100 \\
\hline
\end{tabular}

Highest number of patients 44 (55\%) received dual therapy with inj. Ceftriaxone plus oral Clarithromycin. Dual therapy with oral Coamoxiclav plus oral Clarithromycin was used in $12(15 \%)$ patients. Inj. Ceftriaxone plus Cloxacillin was used in $10(12 \%)$ and inj Ceftriaxone plus oral Linezolid was used in $2(2 \%)$ patients. Monotherapy with inj Ceftriaxone was used in 12 $(15 \%)$ and inj meropenem was used in $2(2 \%)$ of patients. (Table-III)

Table III

Empirical selection of antibiotic

\begin{tabular}{lcccc}
\hline Antibiotic & $\begin{array}{c}\text { Patient } \\
\text { No }\end{array}$ & \multicolumn{3}{c}{ Outcome } \\
& 12 & 15 & 12 & 0 \\
\hline $\begin{array}{l}\text { Inj. Ceftriaxone } \\
\begin{array}{l}\text { Inj. Ceftriaxone plus oral } \\
\text { Clarithromycin }\end{array}\end{array}$ & 44 & 55 & 44 & 0 \\
$\begin{array}{l}\text { Oral co-amoxiclav plus oral } \\
\text { Clarithromycin }\end{array}$ & 12 & 15 & 12 & 0 \\
$\begin{array}{l}\text { Inj. Ceftriaxone plus oral } \\
\text { Cloxacillin }\end{array}$ & 10 & 12 & 10 & 0 \\
$\begin{array}{l}\text { Inj. Ceftriaxone plus oral } \\
\text { Linezolid }\end{array}$ & 2 & 2 & 2 & 0 \\
Inj. Meropenem & 2 & 2 & 2 & 0 \\
\hline Total & 82 & 100 & 82 & 00 \\
\hline
\end{tabular}

Monotherapy with single antibiotic was used in 14 $(18 \%)$ patients, dual therapy with two anti biotic was used in $56(68 \%)$ patients. Triple antibiotic was used in $12(14 \%)$ patients. (Table-IV)

Table IV

Number of antibiotic used for each patient

\begin{tabular}{lcc}
\hline Number of antibiotic used & \multicolumn{2}{c}{ Patient } \\
& No & $\%$ \\
\hline Mono therapy & 14 & 18 \\
Dual therapy & 56 & 68 \\
Triple therapy & 12 & 14 \\
\hline Total & 82 & 100 \\
\hline
\end{tabular}


All the patients including patient with parapneumonic effusion recovered completely. ${ }^{1}$ None of the patients developed lung parenchymal damage or lung abscess. No drug related adverse effects were observed in any patient.

\section{Discussion}

In Bangladesh there is lack of facility of sputum for culture and sensitivity (c/s) test even in teaching hospitals. So CAP is treated by antibiotic selected empirically from the knowledge of experience. In this study we observed the prescription pattern of antibiotic selected on empirical basis in the management of community acquired pneumonia. We evaluated the effectiveness of the antibiotic by monitoring the outcome of treatment. We have gone through a study carried out in medicine ward and pulmonology ward of Bangladesh institute of research and rehabilitation in diabetes, endocrine and metabolic disorders (BIRDEM) in Bangladesh and found that sputum for $\mathrm{c} / \mathrm{s}$ test of CAP patient revealed the organism streptococcus pneumoniae, streptococcus pyogens, staphylococcus aureus, klebsiella pneumoniae. These organisms were sensitive to Ceftriaxone, Meropenem, Co amoxiclav, Clarithromycin and Linezolid. ${ }^{12}$

In a study from China common empirical antibiotic for CAP was monotherapy with Levofloxacin (15\%).13 So there is geographical variation of micro organisms and antibiotic use for CAP treatment. In an American study it is observed that anti biotic treatment for CAP is started by empirical selection and thereafter adjustment of the anti biotic according to the sputum c/s test report is done. They recommended monotherapy with Macrolides or Doxicycline in most patients and Levofloxacin or Moxifloxacin as second line drug. Recommendations for the choice of antibiotic differ among guidelines and among the various subclasses of patients as in out patient care. In patient ward and in ICU patient, superiority of beta lactam and combination of beta lactam plus macrolide is based on cohort studies and observational studies.

Randomized controlled trial evaluates the efficacy of beta lactam antibiotic alone versus beta lactam plus macrolide or versus levofloxacin alone in the treatment of hospitalized CAP. Result showed the superiority of beta lactam in patients having CURB 65 score $0-1$ and 2 . Study describes the superiority of combination drug treatment with beta lactum plus macrolide in patients with more severe form of community acquired pneumonia having CURB 65 score $>3.14$
So empirical antibiotic selection is important for the effective management of CAP in resource poor hospital settings. We used monotherapy in 14 $(18 \%)$ of cases. Dual therapy was used in most of the cases $56(68 \%)$ and triple antibiotic was used in $12(14 \%)$ patients. Multiple antibiotic was used because of inability to identify the organism causing CAP.

There are limitations of this study as it enrolled a small number of patients and it was a single centre study. Intensive care unit (ICU) patients with severe CAP were not included in this study. Multi centre study with large number of patients including ICU patients will reveal a better interpretation regarding the outcome of empirical selection of antibiotic in CAP patients.

\section{Conclusion}

CAP is treated in most of the hospitals, even in large teaching hospitals in Bangladesh by selecting the antibiotic empirically from the previous knowledge and experience of physicians in this geographical area. Although the outcome is good all these attempt can give rise to the development of antibiotic resistance and there is also misuse of antibiotic. So the improvement of hospital laboratories to perform sputum for culture and sensitivily test is necessary.

\section{References}

1. Andrews J, Nadjm B, Grant V, Shetty. N. Community acquired pneumonia. Curr Opin Pulm Med 2003; 9:175-80

2. Postma DF, van Werkhoven $\mathrm{CH}$, van Elden $\mathrm{LJ}$, et al. Antibiotic treatment sWaegy for community acquired pneumonia in adults. N Engl $\mathrm{J}$ Med 2015; 372: 1312-1323

3. Jain S, Self WH, Wunderink RG, Fakhran S, et al. Community acquired pneumonia requiring hospitalization among U.S. adults. N Engl J Med 2015; 373: 415-27

4. Carratala J, Fernandez-Sabe N, Ortega L, et al. Outpatient care compared with hospitalization for community acquired pneumonia: a randomized trial in low risk patients. Ann Intern Med 2005; 142: 165-72

5. Houck PM, Bratzler DW, Nasa W. Ma A, Bartlett JG. Timing of antibiotic administration and outcomes for patients hospitalized with community acquired pneumonia. Arch Intern Med 2004; 164: 637-44 
6. Beovic B, Bonac B, Kese D, Avsic-Zupanc T, et al. Aetiology and clinical presentation of mild community acquired bacterial pneumonia. Eur $\mathrm{J}$ Clin Microbiol Infect Dis 2003; 22: 584-91

7. File TM. Community acquired pneumonia. Lancet 2003; 362: 1991-2001

8. Mokabberi R, Haftbaradaran A, Ravakhah K. Doxycycline vs levofloxacin in the treatment of community acquired pneumonia. J Clin Phrm Ther 2010; 35: 195-200

9. Bauer TT, Ewing S, Marre I, Suttorp N, et al CURB 65 predicts death from community acquired pneumonia. J Intern Med 2006, 260: 93-101

10. Restrepo M 1, Mortensen EM, Velez JA, Frei C,, et al. A comparative study of community acquired pneumonia patients admitted in the ward and the ICU. Chest 2008; 133: 610-617
11. Jefferson $\mathrm{T}$, Rivetti $\mathrm{D}$, Rivetti $\mathrm{A}$, Rudin $\mathrm{M}$, et al. Efficacy and effectiveness of influenza vaccine in elderly people. Lancet 2005; 366: 1165-1174

12. Saibal MAA, Rahman SHZ, Nishat L, Sikder NH, et al. Community acquired pneumonia in diabetic and non diabetic hospitalized patients; presentation, causative pathogens and outcome. Bangladesh Med Res Counc Bull 2012; 38: 98-103

13. Nie XM, Li YS, Yang ZW, et al. Initial empiric therapy for community acquired pneumonia in Chinese hospitals. Clin Microbial Infect 2018; 24: 658-63

14. Lutfiyya MN, Henley E, Chang LF, Reyburn SW. Diagnosis and treatment of community acquired pneumonia. Am Fam Physician 2006; 73: 442-450 